Immediacy Premium: A Simpler Measure to Estimate the Hyperbolic Delay Discounting Rate $(k)$

\author{
Harli R. Berk, Tanya A. Gupta, Federico Sanabria
}

\title{
Arizona State University
}

Harli R. Berk, Arizona State University, Department of Psychology, Tempe, Arizona, United States.

Tanya A. Gupta, Arizona State University, Department of Psychology, Tempe, Arizona, United States.

Federico Sanabria*, Arizona State University, Department of Psychology, Tempe, Arizona, United States. *Corresponding author: PO Box 871104, Tempe, AZ 85287-1104, United States. Federico.Sanabria@asu.edu. 


\begin{abstract}
The estimation of delay discounting rates $(k)$ typically assumes that the relative subjective value of a reinforcer declines as a reciprocal function of its delay. Nonetheless, estimates of $k$ based on least-squares fits of the hyperbolic discounting function to relative subjective values appear to have serious limitations. This curve-fitting method yield average discounting functions that may not accurately reflect the individual subjects' data. The present study used the hyperbolic discounting function to derive a new dependent measure, termed immediacy premium, which is a linear function of delay. By averaging linear rather than reciprocal functions, the averaged estimates obtained from immediacy premiums are more representative of individual data, and comparisons between mean estimates across treatments or samples is more meaningful. Using published data, the similarity of $k$ estimates based on least-square fits to relative subjective values and immediacy premiums was evaluated. The assumptions of this curve-fitting method (normally-distributed error with mean of zero, independent across delays within the same subject) were evaluated for each dependent measure. Systematic species-dependent differences in estimates of $k$ were observed when obtained from one measure or another. Violations of curve-fitting assumptions were observed regardless of measure, but primarily in human data. These violations were simulated to determine the likelihood that they emerge from the transformation between dependent measures. Taken together, the reported analysis yields a qualified support for the adoption of immediacy premiums as the basis for the estimation of $k$.
\end{abstract}

Keywords. Delay discounting, hyperbolic discounting, intertemporal choice, impulsivity, parameter estimation, method of least squares 


\section{Immediacy Premium: A Simpler Measure to Estimate the Hyperbolic Delay Discounting Rate $(k)$}

Delay discounting, or temporal discounting, is the decrease in the value of a reward as the delay to the receipt of that reward increases (Madden \& Bickel, 2010; Odum, 2011; Vanderveldt, Oliveira, \& Green, 2016). Estimates of delay discounting rate are often used to study impulsivity in humans and animals and to mathematically characterize such abstract psychological constructs as willpower and compulsion (Levitt, Sanchez-Roige, Palmer, \& MacKillop, 2020). The accurate estimation of delay discounting rates has important implications for research on psychopathology, prosocial behavior, and other social concerns (Arbuthnott, 2010; da Matta, Gonçalves, \& Bizarro, 2012; Johnson \& Bickel, 2008; Lempert, Steinglass, Pinto, Kable, \& Simpson, 2019).

Delay discounting rates are typically estimated, for each individual subject, on the basis of a series of estimated indifference points that constitute the empirical delay discounting function (Figure 1). An indifference point for an immediate reward is the value of a delayed reward such that the immediate and delayed reward are equally likely to be chosen. The subjective value of a delayed reward is the value of the immediate reward at indifference. The relative subjective value (RSV) of a delayed reward is its subjective value divided by its undiscounted value. For instance, for an individual who is indifferent between $\$ 1,000$ in 12 months and $\$ 625$ now, the subjective value of $\$ 1,000$ in 12 months is $\$ 625$; the RSV of $\$ 1000$ in 12 months is thus $\$ 625 / \$ 1,000=0.625$.

A prevalent model of delay discounting, derived from the matching law (Davison \& McCarthy, 1988) is the hyperbolic discounting function (Ainslie, 2017; Madden \& Bickel, 2010), 


$$
\operatorname{RSV}(D)=(1+k D)^{-1}
$$

where $\operatorname{RSV}(D)$ is the relative subjective value of a reward with delay $D$, discounted at a rate $k$. Equation 1 captures a key feature of empirical discounting data: $R S V$ estimates ( $r s v$ ) typically decline more steeply between shorter delays than between longer delays. Figure 1 shows the RSV predicted by Equation 1 at various delays and two $k$ values.

\section{*** FIGURE 1 ABOUT HERE ***}

In general, the hyperbolic discounting function provides a better fit to empirical data than alternative, single-free-parameter models such as the normative exponential discounting function (Kirby \& Maraković, 1995; Mazur \& Biondi, 2009; McKerchar et al., 2009; but see Luhmann, 2013). The fit of Equation 1 to data improves by raising $D$ (hyperbola-like model) or the whole denominator (hyperboloid model) to the power of a free parameter $s$ (McKerchar et al., 2009). The inclusion of $s$ in the estimation of $k$ involves two important drawbacks: there is no unambiguous interpretation of $s$ and, given the small number of $r s v$ on which estimates of $k$ and $s$ are typically based (rarely more than 6), there is a substantial risk of overfitting.

The estimation of discounting rate $k$ is often based on fitting Equation 1 to an empirical delay discounting function using the method of least squares. This method assumes that

$$
r s v(D)=\left(1+k_{r s v} D\right)^{-1}+\varepsilon_{r s v}
$$


where $\varepsilon_{r s v}$, error variance, is sampled for each probe $D$ from independent normal distributions with mean of zero. A limitation of this method is that the $r s v$ derived from the mean of multiple $k_{r s v}$ estimates may not be representative of the $r s v$ from which each individual $k_{r s v}$ was estimated. Figure 2A illustrates this limitation: note that $r s v$ means and the fit of Equation 1 to those means (" $\times$ " connected by gray lines) do not fall within the range of discounting functions drawn from mean $k_{r s v} \pm 95 \%$ confidence interval (gray band within dashed curves).

*** FIGURE 2 ABOUT HERE ***

A solution to this limitation suggests an alternative method to estimate $k$, which involves a transformation of the RSV,

$$
\mathrm{IP}=(1-\mathrm{RSV}) / \mathrm{RSV} \text {. }
$$

The immediacy premium (IP) of a reward is the ratio of the value lost due to its delay (1 - RSV) to the value preserved despite its delay (RSV). For example, for an individual who is indifferent between $\$ 1,000$ in 12 months and $\$ 625$ now, the IP of the delayed $\$ 1,000$ is the ratio of the $\$ 1,000-\$ 625=\$ 375$ lost due to the 12 -month delay to the $\$ 625$ preserved despite that delay: $\$ 375 / \$ 625=0.600$. That is, for every dollar of value preserved over 12 months, 60 cents are lost over that period of time. An alternative description of this indifference relation is that, barring endowment effects (Marzilli Ericson \& Fuster, 2014), this individual is willing to pay no more than a 60 -cent premium for every delayed dollar that is delivered immediately.

The combination of Equations 1 and 3 reveal that IP is a linear function of $k$, 


$$
I P(D)=k D
$$

Discounting rate $k$ may thus be estimated from observed immediacy premiums (ip) using the method of least squares, assuming that

$$
i p(D)=k_{i p} D+\varepsilon_{i p}
$$

where $\varepsilon_{i p}$ has the same characteristics as $\varepsilon_{r s v}$ in Equation 2. Unlike Equation 2, however, the ip derived from the mean of multiple $k_{i p}$ estimates is the mean ip from which each individual $k_{i p}$ was estimated. Note in Figure 2B that ip means fall close to the range of discounting functions drawn from mean $k_{i p} \pm 95 \%$ confidence interval (gray band within dashed curves), and that the fit of Equation 3 to those means (" $\times$ ") overlaps with the discounting function drawn from mean $k_{i p}$ (solid black line).

Differences between Equations 2 and 5, highlighted in Figure 2, raise the question of whether $k_{r s v}$ and $k_{i p}$ estimates, based on the method of least squares, vary systematically. The present paper verifies this difference, based on empirical reports of individual $r s v$ in the Journal of the Experimental Analysis of Behavior in various species. This paper also examines the relative merit of the RSV-and IP-based methods for estimating $k$, on the basis of whether residuals obtained from the least square error method are (a) centered around zero, (b) normally distributed, and (c) independent across levels of the independent variable (i.e., across delays) (Draper \& Smith, 1998). 


\section{Methods}

Empirical papers were first selected for potential inclusion using the PsycINFO database on January 8, 2018. A search was conducted only within the Journal of the Experimental Analysis of Behavior using the terms "(temporal OR delay) discounting"; this journal was selected because it systematically reports individual-subject data. This search yielded 84 papers. These papers were further examined, keeping only those that contained at least one figure that reported at least 5 indifference points for individual subjects, where each indifference point was reported as a subjective or relative subjective value ( $r s v)$ at a delay. Figures that contained layered data points, such that data could not be reliably extracted, were not considered. This filter narrowed down the initial pool to 7 papers. Table 1 lists the 7 papers included in the analyses along with key characteristics. Pooled across studies, data was extracted from 15 figures representing data from 27 humans, 12 pigeons, and 19 rats. The type of reward, the range of its magnitude, the range of delays, and the methods for estimating indifference points and $k$ varied between studies. Data were extracted from relevant figures using DigitizeIt 2.2.2 (Bormann, 2016). When possible, values on the $x$-axis (delays) were obtained from the methods section in the corresponding paper. These data were then transformed into $r s v$ (if necessary) and ip.

Individual datasets were defined as the set of indifference points for each individual subject in each condition. So, for instance, if a set of indifference points was obtained from one participant using a \$20 larger-later reward, and another set of indifference points was obtained from the same individual using a $\$ 20,000$ larger-later reward, each set counted as an individual dataset. Equations 2 and 5 were fit to each individual dataset using the method of least squares implemented using the Solver add-in of Microsoft @ Excel for Mac 16.2. Estimates of $k\left(k_{r s v}\right.$ and $\left.k_{i p}\right)$ and residuals were further analyzed. In particular, normalized error was estimated from 
residuals obtained from each individual dataset, each divided by its standard deviation in the corresponding dataset (normalized residuals). Normalization was conducted to equate the expected variance of error across estimation methods.

*** TABLE 1 ABOUT HERE ***

\section{Results}

\section{Are estimates of $k$ different when estimated from $r s v$ vs. ip?}

Figure 3 shows the correlation between $k_{r s v}$ and $k_{i p}$ estimates in humans (left panel) and non-human animal subjects (right panel) in log-log scale. In humans, the difference between $k_{r s v}$ and $k_{i p}$ estimates appears to increase as the estimates increase. In addition, $k_{r s v}$ estimates appear to be systematically higher [mean $k_{r s v}-k_{i p}=0.026 \pm 0.010$ days $^{-1} ; t(85)=2.53, p=.013$ ] and more variable (variance of $k_{r s v}=0.040$ days $^{-2}$, variance of $k_{i p}=0.014$ days $^{-2}$ ) than $k_{i p}$ estimates. In animal subjects, mean $k_{r s v}$ estimates appear to be systematically lower than mean $k_{i p}$ estimates. Figure 3 (right panel) shows this difference as a larger number of data points above the identity line. This difference is visible in pigeons [mean $k_{r s v}-k_{i p}=-0.263 \pm 0.057 \mathrm{~s}^{-1} ; t(31)=4.69, p<$ .001 ] but not in rats [mean $k_{r s v}-k_{i p}=-0.041 \pm 0.049 \mathrm{~s}^{-1} ; t(34)=.856, p=.398$ ]. A systematic difference between the variance of $k_{r s v}$ and $k_{i p}$ estimates was observed in pigeons, but not in rats $\left(k_{r s v}\right.$ pigeons $=0.285 \mathrm{~s}^{-2}, k_{i p}$ pigeons $=0.548 \mathrm{~s}^{-2} ; k_{r s v}$ rats $=0.541 \mathrm{~s}^{-2}, k_{i p}$ rats $\left.=0.582 \mathrm{~s}^{-2}\right)$. 


\section{Which method yields mean residuals closer to zero?}

If the mean of $r s v$ and ip samples are the best estimates of the mean $R S V$ and $I P$ in the population, then the residuals from fitting Equations 1 and 4 to data are the best estimates of error in $r s v\left(\varepsilon_{r s v}\right.$ in Equation 2) and ip ( $\varepsilon_{i p}$ in Equation 5) measurement. Figure 4 shows the correlation between normalized residuals obtained from $r s v$ and ip in humans (left panel) and non-human subjects (right panel). Figure 4A (top left panel) shows that the normalized residuals obtained from $r s v$ were on average positive, and those obtained from ip negative (see inset graph). This suggests that hyperbolic functions drawn from $k_{r s v}$ generally overestimated human $r s v$, and hyperbolic functions drawn from $k_{i p}$ generally underestimated human ip. Nonetheless, squared normalized residuals, which measure average distance from origin, were generally smaller when obtained from $r s v($ mean $=1.441 \pm 0.085)$ than when obtained from ip $[2.343 \pm$ $0.229 ; t(539)=3.77, p<.001]$.

\section{*** FIGURE 4 ABOUT HERE ***}

Normalized residuals obtained from animals clustered close to zero than those obtained from humans, regardless of whether they were obtained from $r s v$ or ip (compare Figure 4B to Figure 4A, and corresponding inset graphs). Squared normalized residuals obtained from $r s v$ $($ mean $=0.849 \pm 0.051)$ were similar to those obtained from ip $[$ mean $=0.932 \pm 0.060 ; t(365)=$ $0.575, p=.283]$

\section{Which method yields more normally-distributed residuals?}

Normality of the distribution of residuals was assessed using quantile-quantile (Q-Q) plots, which represent the correlation between (empirical) normalized residuals observed in a 
study and the theoretical (normally distributed) normalized residuals. A theoretical normalized residual is a quantile $q$ of a normal distribution with mean zero and standard deviation of the empirical normalized residuals; the interval between quantiles contains a proportion $1 /(n-1)$ of the distribution, where $n$ is the number of empirical residuals in a study. To the extent that normalized residuals are normally distributed and centered on zero, the data points in the Q-Q plot would fall on the identity line. Distance relative to the identity line was quantified as the sum of the square difference between observed and theoretical normalized residuals.

Figure 5 shows the Q-Q plots for normalized residuals obtained for humans (panel A) and non-human animals (panel B), from $r s v$ and ip. Human data appear to be more layered than animal data (see inset graphs), suggesting more variability in normality among human studies than among animal studies. Human data also suggest that the distribution of residuals was more negatively skewed (long left tail) when obtained from $r s v$, and more positively skewed (right long tail) when obtained from ip. Layering and differences in skewness across methods is not evident in animal data.

\section{*** FIGURE 5 ABOUT HERE ***}

Table 2 shows the sum of the squared distance to the identity line (and index of nonnormality) in humans and non-human subjects, arranged by study. In all human studies, residuals were more normally distributed when obtained from $r s v$ than when obtained from ip. Results from animal studies were more balanced, with more normal residuals when obtained from $r s v$ in 3 of 5 studies. This is reflected in the overlap between Q-Q plots in Figure 5B. 
$* * *$ TABLE 2 ABOUT HERE ***

\section{Are residuals sequentially independent?}

To determine the sequential independence of residuals, lag-1 autocorrelations were conducted on normalized residuals obtained from $r s v$ and ip in each dataset (Hibbs, 1973). Autocorrelation coefficients were obtained from each dataset by correlating each normalized residual at each delay $D$ with the normalized residual at the next delay. Autocorrelation coefficients were squared $\left(r^{2}\right)$ in each dataset, and the difference between $r^{2}$ obtained from $r s v$ and ip $\left(\Delta r^{2}\right)$ served as a measure of relative sequential dependence among residuals. Positive $\Delta r^{2}$ indicate that residuals obtained from $r s v$ are more sequentially dependent than residuals obtained from ip.

Table 3 reports the $\Delta r^{2}$ for each study under consideration. Only two studies showed significant $\Delta r^{2}$; both were positive, indicating that, in those studies, residuals obtained from $r s v$ were more sequentially dependent than those obtained from ip. Both studies were conducted with human participants; no animal study yielded a significant $\Delta r^{2}$, suggesting that concerns of sequential dependency among residuals may be restricted to estimates of $k$ based on human $r s v$.

\section{*** TABLE 3 ABOUT HERE $* * *$}

\section{Monte Carlo Simulations}

So far, the analysis of empirical data suggests a larger and more systematic divergence between $r s v$ - and ip-based methods for estimating $k$ in human data than in animal data. In humans, normalized residuals obtained from ip were farther from zero (Figure 4) and were less 
normally distributed (Table 2) than those obtained from $r s v$. It is possible that this pattern emerges from transforming $r s v$ generated by Equation 2 into ip according to Equation 3. Nonetheless, the normalized residuals obtained from $r s v$ in two human studies (Johnson \& Bickel, 2002; Myerson \& Green, 1995) were more sequentially dependent than those obtained from ip (Table 3). It is possible that this pattern emerges from transforming ip generated by Equation 5 into $r s v$. To evaluate these scenarios, they were simulated using Monte Carlo methods with two values of $k$, sampling $r s v$ and ip error ( $\varepsilon_{r s v}$ and $\varepsilon_{i p}$ from Equations 2 and 5, respectively) from independent normal distributions with mean zero and standard deviation drawn from empirical data. The output of these simulations was then compared to empirical findings.

Two values of $k, 0.001$ and 0.1 days $^{-1}$ (shallow and steep discounting, respectively), were selected to cover a broad range of typical estimates from human data (cf. Figure 3A). Based on empirical data, the standard deviation of $\varepsilon, \sigma(\varepsilon)$, was assumed to be a power function of delay $D$,

$$
\sigma(\varepsilon)=c D^{m}
$$

where $m$ and $\log (c)$ correspond to the slope and $y$-axis intercept of the function in a log-log plot. Figure 6 shows the fits of Equation 6 to the standard deviation of residuals obtained from $r s v$ (“०”) and ip (“×”), on each delay $D$ across datasets within the same human study.

*** FIGURE 6 ABOUT HERE *** 
Two scenarios were evaluated. In the first scenario $(r s v \rightarrow i p), r s v$ were generated from Equation 2 (i.e., including error) for each of 6 delays (1, 10, 50, 300, 1500, 3000 days) covering most of the range of empirical delays (cf. Figure 6). The simulated $r s v$ was then transformed to ip using Equation 3; $k_{i p}$ was recovered by fitting Equation 4 to the ip across delays (slope of fitted linear function, relating ip to delay, that passes through the origin) using the method of least squares. Simulated normalized residuals were obtained from the difference between the ip predicted from the fitted Equation 4 and the simulated $i p$. Thus, in the $r s v \rightarrow i p$ scenario, $k_{i p}$ estimates and their corresponding residuals were obtained from "true" noisy $r s v$.

In the second scenario (ip $\rightarrow r s v$ ), ip were generated from Equation 5 for the same 6 delays. The simulated ip was then transformed to $r s v\left[r s v=(1+i p)^{-1}\right] ; k_{r s v}$ was recovered by fitting Equation 1 to the $r s v$ across delays. Simulated normalized residuals were obtained from the difference between the $r s v$ predicted from the fitted Equation 1 and the simulated $r s v$. Thus, in the ip $\rightarrow r s v$ scenario, $k_{r s v}$ estimates and their corresponding residuals were obtained from "true" noisy ip.

In both scenarios, when adding $\varepsilon$ to a simulated ip or $r s v$ yielded a negative value, it was discarded and a new $\varepsilon$ was sampled. Curve fitting was conducted using the method of least squares. To distinguish the input $k\left(0.001\right.$ or 0.1 days $\left.^{-1}\right)$ from the recovered $k$ (obtained through transformation and curve fitting), the latter will be labeled as $k^{*}$. In each scenario, 10,000 runs of the simulation were conducted per value of $k$ and delay. Simulations were programmed in Mathematica version 12.2 (Wolfram Research, 2020).

\section{Simulating differences in $k$ estimates and normalized residuals}

Table 4 shows three simulation outputs, mean $k^{*}$, mean normalized residual, and mean squared normalized residuals, for each scenario and $k$. These outputs indicate that, if the correct 
method to estimate $k$ is based on $r s v$ ( $r s v \rightarrow$ ip scenario) and Equation 2 is the "true model," using $i p$ would inflate low $k$ estimates $(0.001 \rightarrow 0.004)$ and shrink high $k$ estimates $(0.100 \rightarrow 0.015)$. The inflation effect would have been expressed as data points above the identity line in the lower-left corner of Figure 3A, but such data points were not obtained. The shrinking effect was indeed observed as data points below the identity line in the upper-right corner of Figure $3 \mathrm{~A}$. Interestingly, transforming "true" ip to $r s v$ (ip $\rightarrow r s v$ scenario) does not affect $k$ estimates significantly (i.e., $k^{*}{ }_{r s v} \approx k_{i p}$ ).

\section{*** TABLE 4 ABOUT HERE ***}

As shown in Figure 4A, most empirical normalized residuals obtained from ip were negative and those obtained from $r s v$ were positive. Regardless of $k$, the simulated normalized residuals are not consistent with these observations: The transformation of "true" rsv to ip yield positive, not negative, normalized residuals; the transformation of "true" ip to $r s v$ yield normalized residuals that are much lower than those obtained from empirical $r s v$ (Table 4). Therefore, the systematically negative normalized residuals obtained from empirical ip and the systematically positive normalized residuals obtained from empirical $r s v$, cannot be explained by the mere transformation of one to the other.

Moreover, the simulated squared normalized residuals were much smaller than those obtained from empirical ip and $r s v$, regardless of $k$ (compare against results in Which method yields mean residuals closer to zero? against Table 4). Such discrepancy suggests that the distance between estimated and observed ip and $r s v$ in empirical human data is unlikely to be solely the product of estimating $k$ based on the wrong dependent measure. Nonetheless, the 
smaller squared normalized residuals obtained from estimating $k^{*}{ }_{r s v}$ than $k^{*}{ }_{i p}$ is consistent with empirical data, particularly when $k$ is large. This consistency data suggests that differences in normalized residual size across methods for estimating $k$ may arise from implementing the wrong method. It does not suggest, however, which method is more likely to be correct.

\section{Simulating differences in the normality of residuals}

Figure 7 shows simulated Q-Q plots for each scenario and k, with intervals between quantiles enveloping $0.5 \%$ of simulated residuals. The transformation of "true" $r s v$ to ip yield $k$ dependent deviations from normality in the distribution of residuals: Equation 4 yields a more positively skewed residuals when fit to ip obtained from more steeply discounted $r s v$ (Figure 7B). These results are consistent with the positively skewed distribution of empirical residuals obtained from ip (Figure 5A). The layering of Q-Q plots in Figure 5A thus likely reflects variability in $k$ across studies. The transformation of "true" ip to $r s v$ yield relatively modest deviations from normality in residuals, which also increase with $k$. These results are also consistent with the relative normality of the empirical distribution of residuals obtained from $r s v$ (Figure 5A; Table 2).

\section{*** FIGURE 7 ABOUT HERE ***}

\section{Simulating differences in sequential dependence}

The index of relative sequential dependence, $\Delta r^{2}$, was computed for the output of both simulated scenarios and $k$ values (Table 5). These indices were computed similarly to those of empirical residuals, but assuming that the squared autocorrelation coefficient $\left(r^{2}\right)$ of "true" normalized residuals was zero, because those residuals were sampled from independent normal 
distributions. Therefore, simulated $\Delta r^{2}$ in the $r s v \rightarrow i p$ scenario were equal to $-r^{2}$ of residuals obtained from simulated ip $\left(\Delta r^{2}=0-r^{2}\right)$, and simulated $\Delta r^{2}$ in the $i p \rightarrow r s v$ scenario were equal to $r^{2}$ of residuals obtained from simulated $r s v\left(\Delta r^{2}=r^{2}-0\right)$.

\section{*** TABLE 5 ABOUT HERE ***}

Regardless of scenario or $k$ value, transforming one dependent measure with uncorrelated error to the other dependent measure-rsv to ip or ip to $r s v$-yielded positively correlated residuals in the latter. In the empirical data, only two human studies display a substantially stronger autocorrelation among residuals obtained from one dependent measure or the other, indicating that residuals obtained from $r s v$ are more sequentially dependent than those obtained from ip in both studies (Table 3). The output of the Monte Carlo simulation suggests that, at least for those two studies, $k$ is more accurately estimated from ip than from $r s v$.

\section{Discussion}

Estimates of $k$ varied systematically depending on whether they are obtained from fitting the hyperbolic decay model to relative subjective values ( $r s v$; Equation 1) or to immediacy premiums (ip; Equation 3), using the method of least square errors (Figure 3). This effect is more noticeable in humans and pigeons than in rats. The direction of this difference in estimates of $k$ appears to depend on the species under study: whereas in humans, particularly those who discount delayed rewards more steeply, estimates of $k$ obtained from $r s v\left(k_{r s v}\right)$ appear to be higher than those obtained from ip $\left(k_{i p}\right)$, the opposite was observed in pigeons. A simulation based on human data suggests that some of these differences are more likely to appear when ip with independent normally distributed error centered on zero are transformed into $r s v$, than vice 
versa (Table 4). Therefore, the relation between $k_{r s v}$ and $k_{i p}$ suggest that ip meet the assumptions of the least square method for the estimation of $k$ better than $r s v$, and should be favored for that purpose.

A close examination of normalized residuals suggests that assumptions of normality and independence were likely met in the case of non-human animals (Figure 5B, Table 3), regardless of whether $k$ was estimated from $r s v$ or ip. It is important to note, however, that mean normalized residuals obtained from $r s v$ were slightly but systematically smaller than those obtained from ip (Figure 4B).

In the case of humans, normalized residuals were somewhat skewed depending on whether they were obtained from $r s v$ or ip (Figure 5A), and in both cases their means were systematically non-zero (Figure 4A). More specifically, fits of Equation 1 to $r s v$ systematically overestimated $r s v$, whereas fits of Equation 4 to ip systematically underestimated $i p$. This difference in the sign of residuals is not surprising, given the inverse relation between RSV and IP (Equation 3). Like in non-human animals, the mean of normalized residuals obtained from $r s v$ were systematically smaller than those obtained from ip, suggesting that the violation of the distributional assumption of mean residuals is less extreme when $k$ is estimated from $r s v$ than from ip. Indeed, simulated transformations from $r s v$ to ip and from ip to $r s v$ yield distributional properties that resemble the empirical data (compare Figures 5A and 7B). Therefore, the relative normality of empirical $r s v$ residuals may emerge from ip with normally-distributed error. This possibility undercuts the ostensive advantage that the normality of $r s v$ residuals confers to an RSV-based method of $k$ estimation. 
Finally, in only two studies, both with humans participants (Johnson \& Bickel, 2002; Myerson \& Green, 1995), residuals were substantially more autocorrelated when data were represented as $r s v$ than as ip. Such difference appears in simulated residuals obtained from ip and transformed to $r s v$ (Table 5). Simulated residuals suggest that the opposite transformation also yields sequentially dependent residuals, but such dependency was not systematically observed in the empirical data. Therefore, it appears that the violation of the independence assumption observed in these studies may have been less likely if $k$ had been estimated from ip and not from $r s v$.

Taken together, the analysis of published empirical data of individual subjects and the simulation of human empirical data suggest that, when $k$ is estimated from human participants, the method of least squares may be more adequately applied to ip than to $r s v$ data. In contrast, it appears that ip and $r s v$ obtained from animal data meet equally well the assumptions necessary to estimate $k$ using the method of least squares. Our recommendation, therefore, is to adopt ip as the dependent measure for studies of intertemporal choice aimed at estimating $k$, especially if estimates are based on the method of least squares. Immediacy premiums may be computed directly from indifference points obtained through traditional methods, simply dividing the difference between undiscounted and discounted value by the discounted value. This measure has the added value that, because it is a linear function of $k$, predictions drawn from the average $k$ estimate match the average predictions drawn from multiple $k$ estimates (Figure 2).

Although the evidence favors the adoption of ip over $r s v$, it also shows some limitations of the former measure. In particular, the extent to which error in human ip measurement is normally distributed and centered on zero is not clear. The positive skew of empirical ip residuals suggests that an analysis based on log-transformed ip may aid in normalizing error 
variance. Interestingly, log-transformed $I P(D)$ has a simple relation with $R S V(D)$ derived from Equations 1 and 4,

$$
\log [I P(D)]=\log (k D) \approx \log (1+k D)=-\log [R S V(D)]
$$

Equation 7 indicates that, to the extent that $k$ or $D$ are high, estimates of $k$ would be very similar regardless of whether they are based on $\log (i p)$ or $\log (r s v)$. Equation 7 thus supports expressing $D$ in small units (e.g., days instead of weeks) to raise $D$ and make the choice of dependent measure irrelevant. It is likely, however, that $k$ estimates will compensate for this change. Moreover, ip computed from indifference points may be negative if the discounted value is greater than the undiscounted value - if delay somehow added value to the reward. In such case, which was reported in several of the studies analyzed here, $\log (i p)$ is undefined; $\log (r s v)$ is simply positive.

In short, the analysis presented here raises critical questions about the adequacy of the prevalent method for estimating discounting rate $k$. Estimations based on a common dependent measure such as $r s v$, particularly those obtained from humans, may be substantially improved with a simple transformation (Equation 3). Such improvement may reduce the likelihood of extremely high estimates (Figure 3A), perhaps enhancing the power of statistical analyses. 


\section{References}

Ainslie, G. (2017). De Gustibus Disputare: Hyperbolic delay discounting integrates five approaches to impulsive choice. Journal of Economic Methodology, 24(2), 166-189. https://doi.org/10.1080/1350178X.2017.1309373

Arbuthnott, K. D. (2010). Taking the long view: Environmental sustainability and delay of gratification. Analyses of Social Issues and Public Policy, 10(1), 4-22. https://doi.org/10.1111/j.1530-2415.2009.01196.x

Bormann, I. (2016). DigitizeIt (version 2.2.2). Brunswick, Germany. Retrieved from www.digitizeit.xyz

da Matta, A., Gonçalves, F. L., \& Bizarro, L. (2012). Delay discounting: Concepts and measures. Psychology and Neuroscience, 5(2), 135-146. https://doi.org/10.3922/j.psns.2012.2.03

Davison, M., \& McCarthy, D. (1988). The matching law: A research review. Hillsdale, NJ: Lawrence Erlbaum Associates.

Draper, N. R., \& Smith, H. (1998). Applied regression analysis. Applied Regression Analysis. New York, NY: John Wiley \& Sons.

Green, L., Myerson, J., Holt, D. D., Slevin, J. R., \& Estle, S. J. (2004). Discounting of delayed food rewards in pigeons and rats: Is there a magnitude effect? Journal of the Experimental Analysis of Behavior, 81(1), 39.

Green, L., Myerson, J., Oliveira, L., \& Chang, S. E. (2013). Delay discounting of monetary rewards over a wide range of amounts. Journal of the Experimental Analysis of Behavior, 100(3), 269-281. https://doi.org/10.1002/jeab.45

Green, L., Myerson, J., Oliveira, L., \& Chang, S. E. (2014). Discounting of delayed and probabilistic losses over a wide range of amounts. Journal of the Experimental Analysis of 
Behavior, 101(2), 186-200. https://doi.org/10.1002/jeab.56

Hibbs, D. A. (1973). Problems of statistical estimation and causal inference in time-series regression models. Sociological Methodology, 5, 252-308. Retrieved from https://www.jstor.org/stable/270839

Johnson, M. W., \& Bickel, W. K. (2002). Within-subject comparison of real and hypothetical money rewards in delay discounting. Journal of the Experimental Analysis of Behavior, 77(2), 129-146. https://doi.org/10.1901/jeab.2002.77-129

Johnson, M. W., \& Bickel, W. K. (2008). An algorithm for identifying nonsystematic delaydiscounting data. Experimental and Clinical Psychopharmacology, 16(3), 264-274. https://doi.org/10.1037/1064-1297.16.3.264

Kirby, K. N., \& Maraković, N. N. (1995). Modeling myopic decisions: Evidence for hyperbolic delay-discounting within subjects and amounts. Organizational Behavior and Human Decision Processes, 64(1), 22-30.

Lempert, K. M., Steinglass, J. E., Pinto, A., Kable, J. W., \& Simpson, H. B. (2019). Can delay discounting deliver on the promise of RDoC? Psychological Medicine, 49(2), 190-199. https://doi.org/10.1017/S0033291718001770

Levitt, E., Sanchez-Roige, S., Palmer, A. A., \& MacKillop, J. (2020). Steep discounting of future rewards as an impulsivity phenotype: A concise review. Current Topics in Behavioral Neurosciences, 47, 113-138. https://doi.org/10.1007/7854_2020_128

Luhmann, C. C. (2013). Discounting of delayed rewards is not hyperbolic. Journal of Experimental Psychology: Learning Memory and Cognition, 39(4), 1274-1279. https://doi.org/10.1037/a0031170

Madden, G. J., \& Bickel, W. K. (Eds.). (2010). Impulsivity: The behavioral and neurological 
science of discounting. Washington, DC: American Psychological Association.

Marzilli Ericson, K. M., \& Fuster, A. (2014). The endowment effect. Annual Review of

Economics, 6, 555-579. https://doi.org/10.1146/annurev-economics-080213-041320

Mazur, J. E., \& Biondi, D. R. (2009). Delay-amount tradeoffs in choices by pigeons and rats: Hyperbolic versus exponential discounting. Journal of the Experimental Analysis of Behavior, 91(2), 197-211. https://doi.org/10.1901/jeab.2009.91-197

McKerchar, T. L., Green, L., Myerson, J., Pickford, T. S., Hill, J. C., \& Stout, S. C. (2009). A comparison of four models of delay discounting in humans. Behavioural Processes, 81(2), 256-259. https://doi.org/10.1016/j.beproc.2008.12.017

Myerson, J., \& Green, L. (1995). Discounting of delayed rewards: Models of individual choice. Journal of the Experimental Analysis of Behavior, 64(3), 263-276. https://doi.org/10.1901/jeab.1995.64-263

Odum, A. L. (2011). Delay discounting: I'm a K, you're a K. Journal of the Experimental Analysis of Behavior, 96(3), 427-439. https://doi.org/10.1901/jeab.2011.96-423

Oliveira, L., Green, L., \& Myerson, J. (2014). Pigeons’ delay discounting functions established using a concurrent-chains procedure. Journal of the Experimental Analysis of Behavior, 102(2), 151-161. https://doi.org/10.1002/jeab.97

Stein, J. S., Pinkston, J. W., Brewer, A. T., Francisco, M. T., \& Madden, G. J. (2012). Delay discounting in Lewis and Fischer 344 rats: Steady-state and rapid-determination adjustingamount procedures. Journal of the Experimental Analysis of Behavior, 97(3), 305-321. https://doi.org/10.1901/jeab.2012.97-305

Vanderveldt, A., Oliveira, L., \& Green, L. (2016). Delay discounting: Pigeon, rat, human-does it matter? Journal of Experimental Psychology: Animal Learning and Cognition, 42(2), 141- 
162. https://doi.org/10.1037/xan0000097

Wolfram Research. (2020). Mathematica. Champaign, IL: Wolfram Research, Inc. Retrieved from https://www.wolfram.com/mathematica 


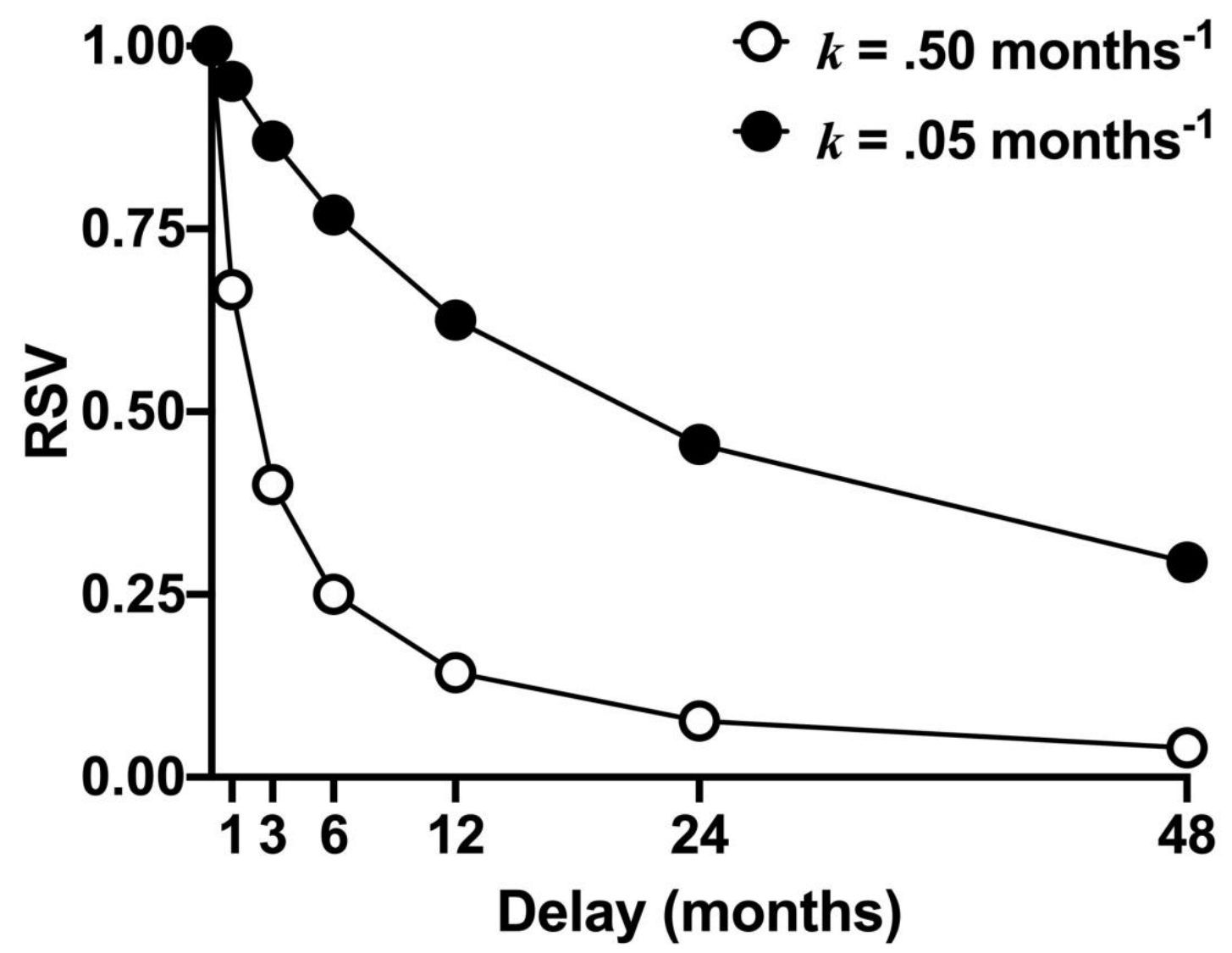

Fig. 1. Hypothetical relative subjective values (RSV) obtained from a hyperbolic discounting function (Equation 1) with discounting rate $k=0.50$ months $^{-1}$ (open circles, steep discounting) and $k=0.05$ months $^{-1}$ (closed circles, shallow discounting). 

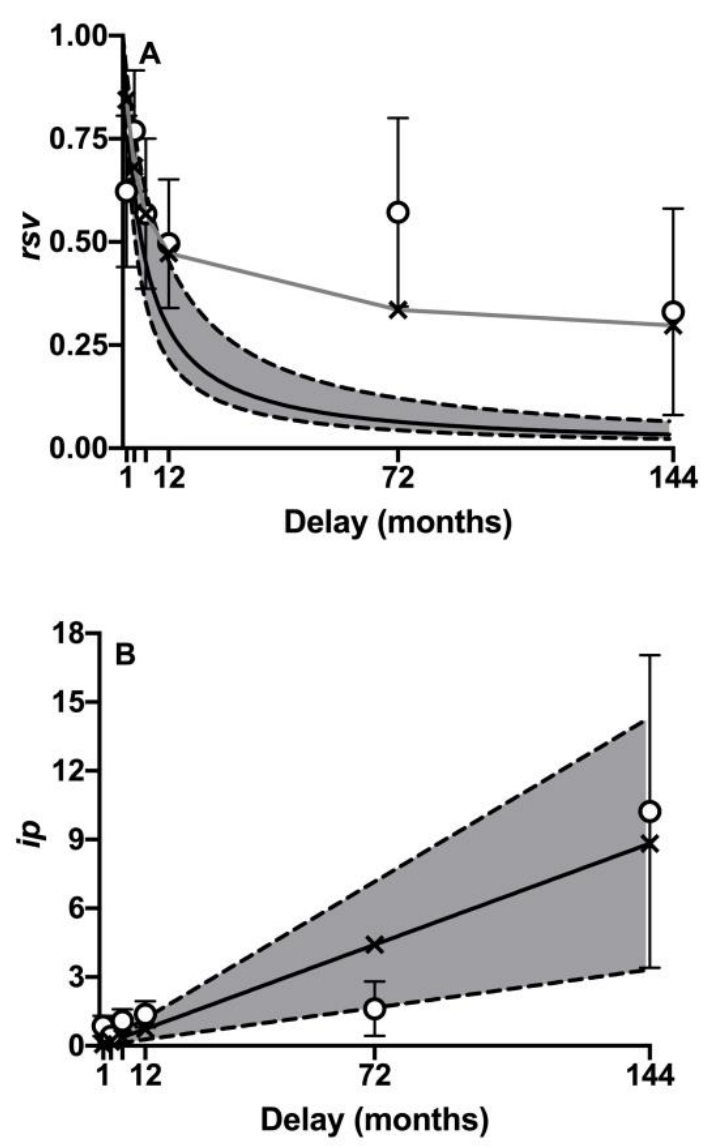

Fig. 2. (A) Mean ( \pm SEM) observed $r s v$ (circles) obtained from individual datasets in the $\$ 250$ reward condition reported in Figure 3 of Green, Myerson, Oliveira and Chang (2014). The “ $\times$ ” connected by gray lines is the best fit of Equation 2 to $r s v$ means, using the method of least squares. The gray band within dashed curves represent the $95 \%$ confidence interval enveloping the discounting functions drawn from individual $k_{r s v}$ estimates (mean $=0.203$ months $^{-1}$, represented as solid black curve; 95\% CI $= \pm 0.103$ months $^{-1}$ ). (B) Representation of the data in panel A as immediacy premiums (ip; Equation 5). The best least-squares fit of Equation 3 to mean ip is only visible as " $\times$ ", because it coincides with the trace of the mean $k_{i p}$ estimate $(0.061$ months $^{-1}$, represented as solid black line; $95 \% \mathrm{CI}= \pm 0.038$ months $^{-1}$, represented as gray band within dashed lines). 

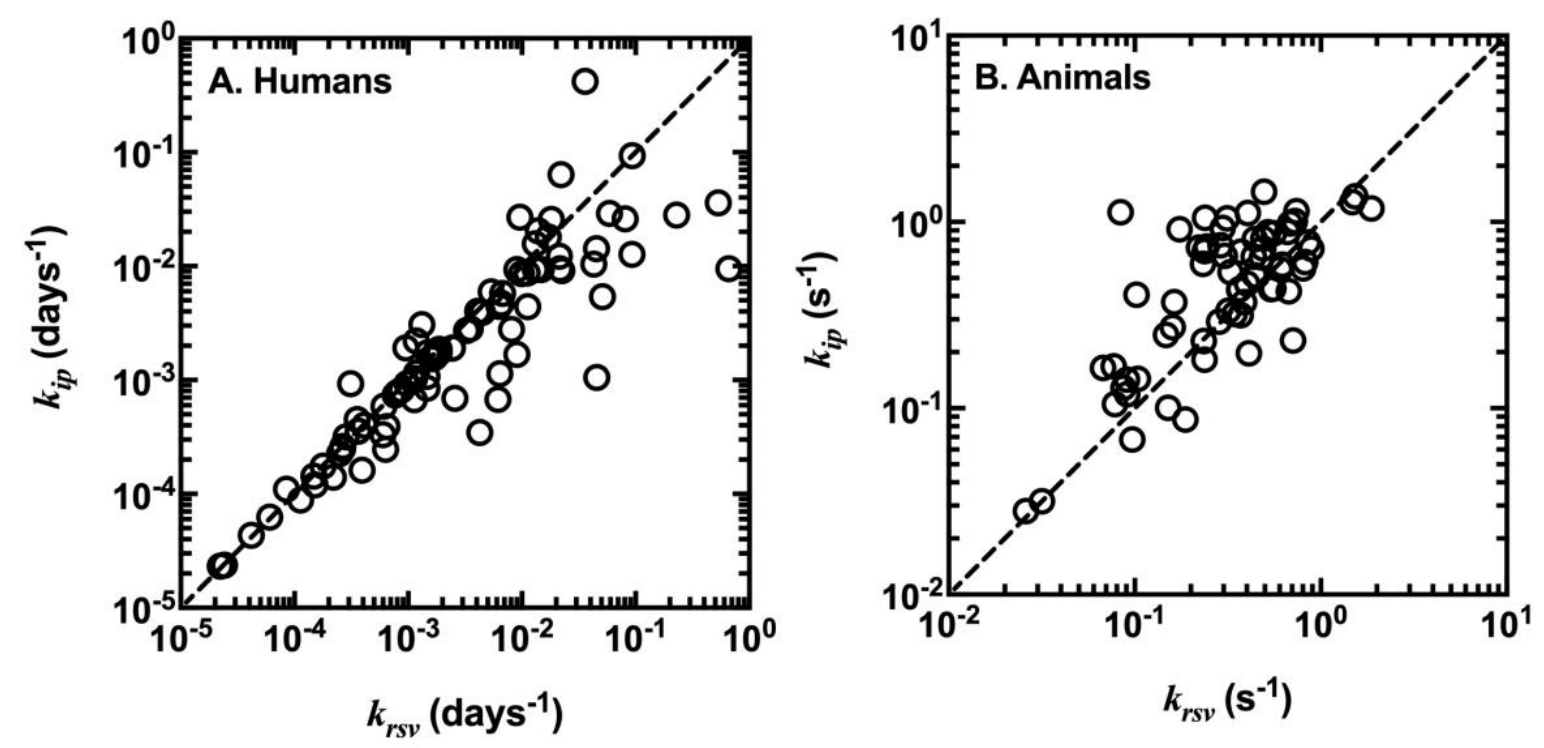

Fig. 3. Delay discounting rate $(k)$ estimates obtained from fitting Equations 1 (RSV, $x$-axis in log scale) and 4 (IP, $y$-axis in log scale) to human (A) and non-human animal (B) data. Identity lines are dashed. 


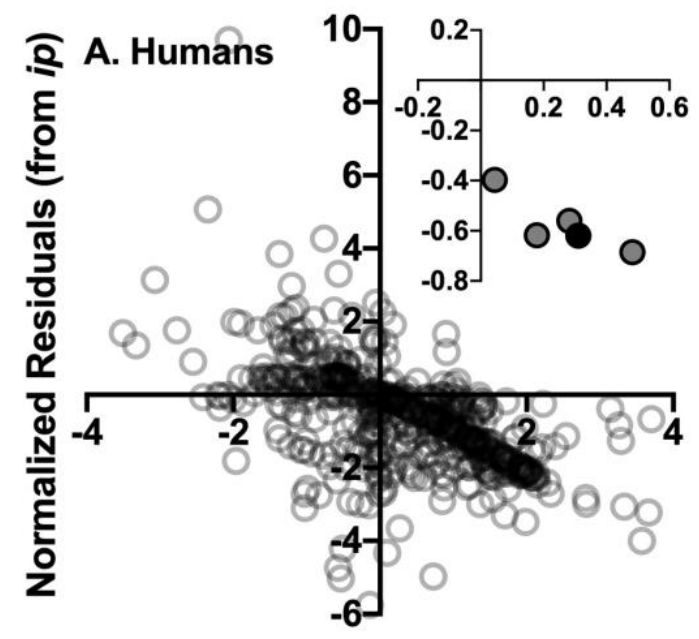

Normalized Residuals (from rsv)

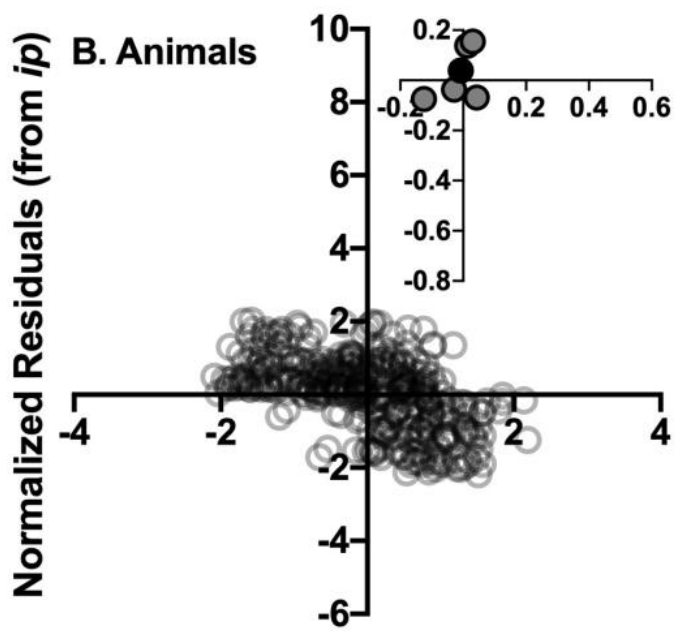

Normalized Residuals (from rsv)

Fig. 4. Normalized residuals (divided by their standard deviation in own dataset) obtained from fitting Equations $1(R S V ; x$-axis) and 3 (IP; $y$-axis) to human (A) and animal (B) data. Inset graphs show the corresponding normalized residuals averaged within each study (gray symbols) and pooled across studies (closed symbols). 

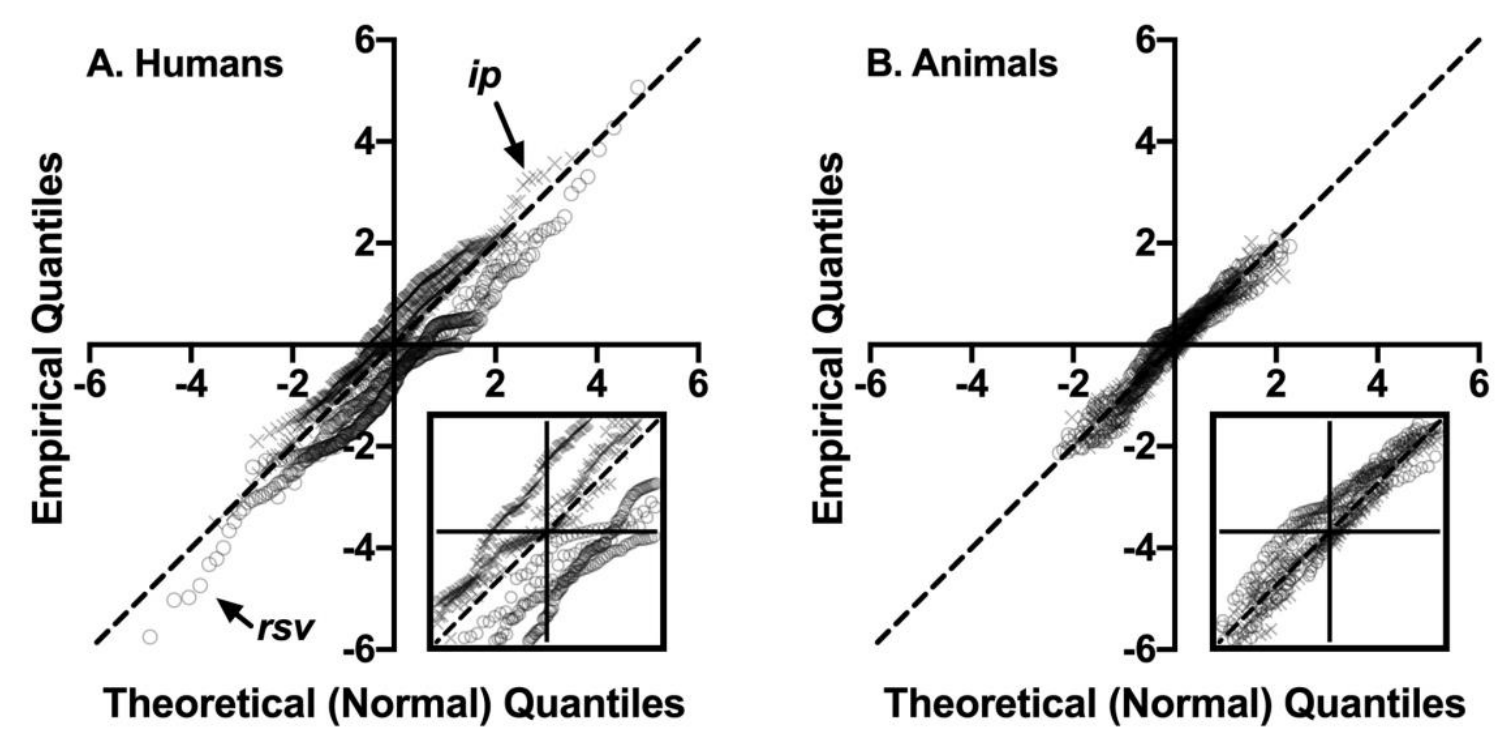

Fig. 5. Q-Q plots of normalized residuals for humans (A) and animals (B) obtained from $r s v$ (“०”) and ip ("×”). Empirical normalized residuals are in the $y$-axis and their normallydistributed correlates are in the $x$-axis; the identity line indicates perfect normality. The inset graphs enlarge the square area between -1 and +1 in both $x$ - and $y$-axes, to better visualize the layering of Q-Q plots. 


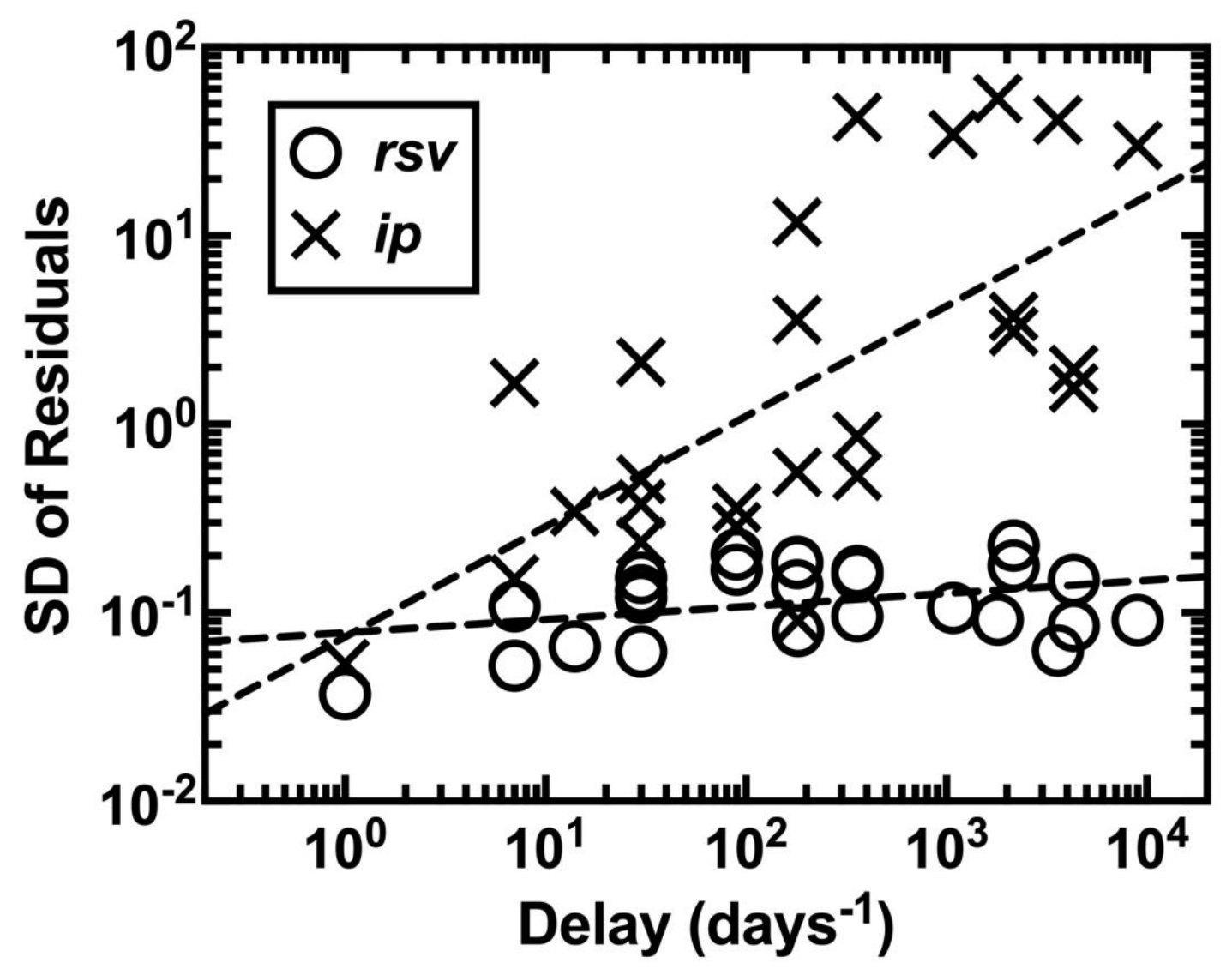

Fig. 6. Standard deviation of normalized residuals obtained from human $r s v$ (" $\circ ")$ and $i p$ (" $\times ”)$ as a function of delay, plotted on a log-log scale. Dashed lines are fits of a power function (Equation 6) to data from $r s v(m=0.069, c=0.078)$ and $i p(m=0.585, c=0.075)$. 


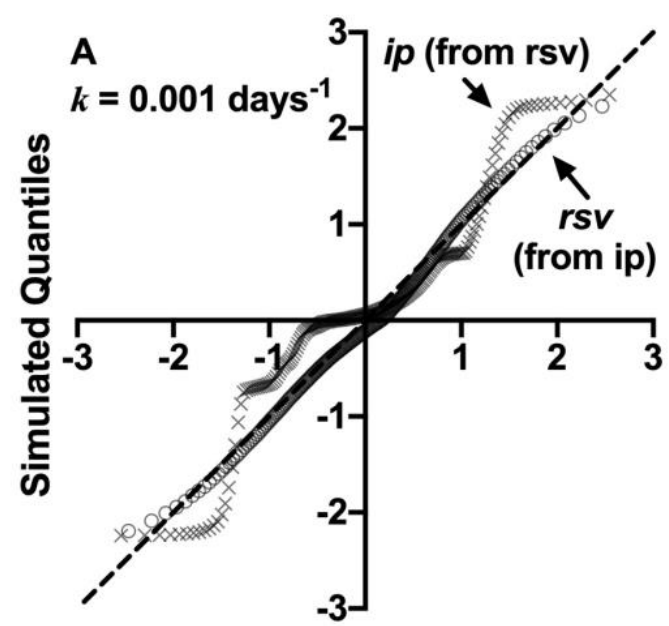

Theoretical (Normal) Quantiles

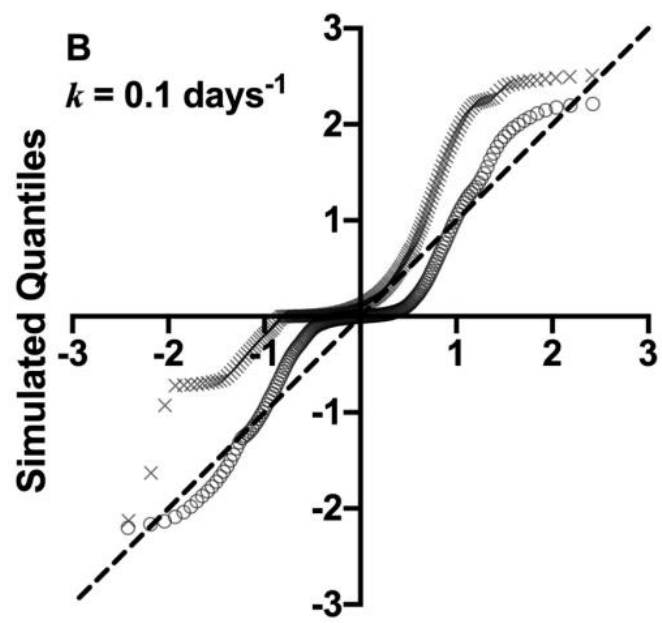

Theoretical (Normal) Quantiles

Fig. 7. Q-Q plots of normalized residuals obtained from simulated human $r s v$ (“०”) and ip (“×”). Simulated residuals were obtained from generating one dependent measure ( $r s v$ from Equation 2, ip from Equation 5) using one of two values of $k\left(0.001\right.$ days $^{-1}$ in $\mathrm{A}, 0.1$ days $^{-1}$ in $\left.\mathrm{B}\right)$, transforming it to the other measure, and fitting the corresponding equation. The identity line indicates perfect normality. 


\section{Table 1}

Sources of data for analysis

\begin{tabular}{|c|c|c|c|c|c|c|}
\hline Study & $\begin{array}{l}\text { Figures } \\
\text { included }\end{array}$ & $\begin{array}{c}\text { Species and } \\
\text { number of } \\
\text { subjects }\end{array}$ & Delays & Amounts & Model used to estimate $k$ & $\begin{array}{c}\text { RSV estimation } \\
\text { procedure }\end{array}$ \\
\hline \multicolumn{7}{|c|}{ Human Participants } \\
\hline $\begin{array}{l}\text { Green et } \\
\text { al. (2013) }\end{array}$ & Fig. 4 & $\begin{array}{l}\text { Humans } \\
6\end{array}$ & $\begin{array}{l}\text { Months: } 1 \text {, } \\
3,6,12,72 \\
144\end{array}$ & $\begin{array}{c}\$ 20 ; \$ 250 ; \$ 3,000 \\
\$ 20,000 ; \$ 50,000 \\
\$ 100,000 ; \$ 500,000 \\
\$ 2,000,000 ; \$ 10,000,000\end{array}$ & $\begin{array}{l}\text { Hyperboloid, Quasi- } \\
\text { Hyperbolic, and Double } \\
\text { Exponential }\end{array}$ & $\begin{array}{l}\text { Adjusting-Amount } \\
\text { Procedure }\end{array}$ \\
\hline $\begin{array}{l}\text { Green et } \\
\text { al. }(2014)\end{array}$ & Fig. 3 & $\begin{array}{l}\text { Humans } \\
3\end{array}$ & $\begin{array}{l}\text { Months: } 1 \\
3,6,12,72 \\
144\end{array}$ & $\begin{array}{c}\$ 20 ; \$ 250 ; \$ 3,000 \\
\$ 20,000 ; \$ 50,000 ; \\
\$ 100,000 ; \$ 500,000\end{array}$ & Hyperboloid & $\begin{array}{l}\text { Adjusting-Amount } \\
\text { Procedure }\end{array}$ \\
\hline $\begin{array}{l}\text { Johnson } \\
\text { \& Bickel } \\
(2002)\end{array}$ & Fig. 1-4 & $\begin{array}{l}\text { Humans } \\
6\end{array}$ & $\begin{array}{l}\text { Days: } 1,7, \\
14,30,182\end{array}$ & $\$ 10 ; \$ 25 ; \$ 100 ; \$ 250$ & $\begin{array}{l}\text { Hyperbolic and } \\
\text { Exponential Decay }\end{array}$ & $\begin{array}{l}\text { Adjusting-Amount } \\
\text { Procedure }\end{array}$ \\
\hline $\begin{array}{l}\text { Myerson } \\
\text { \& Green } \\
(1995)\end{array}$ & Fig. $2 \& 3$ & $\begin{array}{l}\text { Humans } \\
12\end{array}$ & $\begin{array}{c}\text { Days: } 7,30 \\
180,360 \\
1080,3600 \\
9000\end{array}$ & $\$ 1,000 ; \$ 10,000$. & $\begin{array}{l}\text { Hyperbola-like and } \\
\text { Exponential Decay }\end{array}$ & Fixed-Amount Procedure \\
\hline
\end{tabular}




\section{Non- Human Subjects}

\begin{tabular}{|c|c|c|c|c|c|c|}
\hline $\begin{array}{l}\text { Green et } \\
\text { al. (2004) }\end{array}$ & Fig. $1 \& 2$ & $\begin{array}{c}\text { Pigeons } \\
4\end{array}$ & $\begin{array}{l}\text { Seconds: } 1 \text {, } \\
2,4,8,16, \\
32\end{array}$ & $5,12,20$, and 32 pellets & $\begin{array}{c}\text { Hyperbolic and } \\
\text { Hyperbola-like Decay }\end{array}$ & $\begin{array}{l}\text { Adjusting-Amount } \\
\text { Procedure }\end{array}$ \\
\hline $\begin{array}{l}\text { Green et } \\
\text { al. (2004) }\end{array}$ & Fig. $1 \& 2$ & $\begin{array}{c}\text { Rats } \\
4\end{array}$ & $\begin{array}{l}\text { Seconds: } 1 \text {, } \\
2,4,8,16, \\
32\end{array}$ & 5,12 , and 20 pellets & $\begin{array}{c}\text { Hyperbolic and } \\
\text { Hyperbola-like Decay }\end{array}$ & $\begin{array}{l}\text { Adjusting-Amount } \\
\text { Procedure }\end{array}$ \\
\hline $\begin{array}{l}\text { Oliveira } \\
\text { et al. } \\
(2014)\end{array}$ & Fig. 1 & $\begin{array}{l}\text { Pigeons } \\
8\end{array}$ & $\begin{array}{l}\text { Seconds: } 1 \text {, } \\
3,6,10,20\end{array}$ & 16 and 32 pellets & Hyperbolic Decay & $\begin{array}{l}\text { Concurrent-Chain } \\
\text { Adjusting-Amount } \\
\text { Procedures }\end{array}$ \\
\hline $\begin{array}{l}\text { Stein et } \\
\text { al. }(2012)\end{array}$ & Fig. 1 & $\begin{array}{c}\text { Rats (Lewis) } \\
8\end{array}$ & $\begin{array}{c}\text { Seconds: } 0 \text {, } \\
1.25,2.5,5 \text {, } \\
10\end{array}$ & 10 food pellets & Hyperbolic Decay & $\begin{array}{l}\text { Rapid-Determination and } \\
\text { Steady-State Procedures }\end{array}$ \\
\hline $\begin{array}{l}\text { Stein et } \\
\text { al. (2012) }\end{array}$ & Fig. 1 & $\begin{array}{c}\text { Rats } \\
\text { (Fischer) } \\
8\end{array}$ & $\begin{array}{c}\text { Seconds: } 0 \text {, } \\
1.25,2.5,5 \text {, } \\
10\end{array}$ & 10 food pellets & Hyperbolic Decay & $\begin{array}{l}\text { Rapid-Determination and } \\
\text { Steady-State Procedures }\end{array}$ \\
\hline
\end{tabular}




\section{Table 2}

Sum of square distance between observed and theoretical normalized residuals

\begin{tabular}{lcc}
\hline Study & $r s v$ & ip \\
\hline \multicolumn{1}{c}{ Human Participants } & & \\
Green et al. (2013) & $0.619^{*}$ & 12.556 \\
Green et al. (2014) & $3.681^{*}$ & 12.887 \\
Johnson et al. (2002) & $61.533^{*}$ & 126.650 \\
Myerson et al. (1995) & $3.500^{*}$ & 35.569 \\
$\quad$ Non-Human Subjects & & $6.022^{*}$ \\
Green et al. (2004) Pigeons & 7.630 & 3.658 \\
Green et al. (2004) Rats & $2.627^{*}$ & 3.086 \\
Oliveira et al. (2014) Pigeons & $1.709^{*}$ & 1.563 \\
Stein et al. (2012) Rats (Lewis) & $0.525^{*}$ & $1.959^{*}$ \\
Stein et al. (2012) Rats (Fisher) & 2.040 & \\
\hline
\end{tabular}

Note: *Value closer to zero. 


\section{Table 3}

Relative sequential dependence of normalized residuals

Study $\quad$ Mean $\Delta r^{2}(+/-95 \%$ CI $)$

\section{Human Participants}

Green et al. (2013)

$0.01(0.12)$

Green et al. (2014)

$-0.07(0.28)$

Johnson et al. (2002)

$0.07 *(0.06)$

Myerson et al. (1995)

$0.18 *(0.10)$

\section{Non- Human Subjects}

Green et al. (2004) Pigeons

$0.06(0.15)$

Green et al. (2004) Rats

$-0.08(0.19)$

Oliveira et al. (2014) Pigeons

$-0.05(0.12)$

Stein et al. (2012) Rats (Lewis)

$-0.03(0.18)$

Stein et al. (2012) Rats (Fisher)

$-0.13(0.18)$

Note: *Absolute mean $\Delta r^{2}$ greater than its $95 \%$ CI. Positive $\Delta r^{2}$ indicate that normalized residuals are more sequentially dependent when obtained from $r s v$ than from ip. 


\section{Table 4}

Monte Carlo simulation output: $k^{*}$ and normalized residuals

\begin{tabular}{|c|c|c|c|}
\hline & $\begin{array}{c}k^{*} \\
\operatorname{Mean}( \pm 95 \% \mathrm{CI})\end{array}$ & $\begin{array}{c}\text { Mean Normalized } \\
\text { Residuals }( \pm 95 \% \text { CI) }\end{array}$ & $\begin{array}{c}\text { Mean Squared } \\
\text { Normalized Residuals } \\
( \pm 95 \% \text { CI })\end{array}$ \\
\hline \multicolumn{4}{|c|}{$r s v \rightarrow i p$ Scenario $\left(k_{i p}\right)$} \\
\hline$k_{r s v}=0.001$ & $0.004( \pm<0.001)$ & $0.099( \pm 0.009)$ & $0.986( \pm 0.034)$ \\
\hline$k_{r s v}=0.1$ & $0.015( \pm 0.003)$ & $0.488( \pm 0.008)$ & $1.118( \pm 0.037)$ \\
\hline \multicolumn{4}{|c|}{$i p \rightarrow r s v$ Scenario $\left(k^{*} r v\right)$} \\
\hline$k_{i p}=0.001$ & $0.006( \pm 0.011)$ & $-0.047( \pm 0.008)$ & $0.919( \pm 0.023)$ \\
\hline$k_{i p}=0.1$ & $0.100( \pm<0.001)$ & $0.015( \pm 0.008)$ & $0.884( \pm 0.027)$ \\
\hline
\end{tabular}




\section{Table 5}

Monte Carlo simulation output: $\Delta r^{2}$

$$
\Delta r^{2}
$$

Mean $( \pm 95 \% \mathrm{CI})$

\begin{tabular}{ll}
$\boldsymbol{r s v} \boldsymbol{\rightarrow} \rightarrow \boldsymbol{i p}$ Scenario $\left(\boldsymbol{k}^{*} \boldsymbol{i p}\right)$ & \\
$k_{r s v}=0.001$ & $-0.196( \pm 0.003)$ \\
$k_{r s v}=0.1$ & $-0.084( \pm 0.002)$ \\
\hline $\boldsymbol{i p} \rightarrow \boldsymbol{r s v} \boldsymbol{S c e n a r i o}\left(\boldsymbol{k}^{*} \boldsymbol{r}_{s v}\right)$ & $0.153( \pm 0.004)$ \\
$k_{i p}=0.001$ & $0.421( \pm 0.007)$ \\
$k_{i p}=0.1$ &
\end{tabular}

